\title{
Weed Management in Alfalfa -- 20051
}

\section{J.A. Ferrell ${ }^{2}$}

Table 1. Weed Management in Alfalfa.

\begin{tabular}{|c|c|c|}
\hline $\begin{array}{l}\text { Trade Name and } \\
\text { Broadcast Rate/Acre of } \\
\text { Commercial Product }\end{array}$ & Common Name & Remarks \\
\hline \multicolumn{3}{|c|}{ Preplant Incorporated (PPI) } \\
\hline $\begin{array}{l}\text { Balan 60DF } \\
2 \mathrm{lb}\end{array}$ & benefin & \multirow{2}{*}{$\begin{array}{l}\text { Incorporate } 2-3 \text { inches deep before planting for grass control. EPTC } \\
\text { may provide better broad spectrum weed control. Some epinastic } \\
\text { injury to alfalfa usually occurs from EPTC, however, injury is } \\
\text { normally temporary. Do not use if grain or grass crop is to be planted } \\
\text { with alfalfa. Research has shown significant injury as stand reduction } \\
\text { from Balan. Injury would be expected to be less when used on fall } \\
\text { than on spring planted alfalfa. The yield of the first cutting may be } \\
\text { reduced when treated with Balan }\end{array}$} \\
\hline $\begin{array}{l}\text { Eptam } \\
3.5 \mathrm{pt}\end{array}$ & EPTC & \\
\hline \multicolumn{3}{|c|}{ Postemergence - Seedling Alfalfa (First Season) } \\
\hline $\begin{array}{l}\text { Butyrac } 200 \\
\text { or } \\
\text { Butoxone } \\
\text { (1.75 lb gal) } \\
2 \text { to } 3 \text { qts }\end{array}$ & $2,4-\mathrm{DB}$ & $\begin{array}{l}\text { Apply in fall or spring after alfalfa has two to four trifoliate leaves. } \\
\text { Controls emerged annual broadleaf weeds less than } 3 \text { inches tall. Do } \\
\text { not graze or cut for hay for } 60 \text { days after application. Rainfall or } \\
\text { irrigation within } 7 \text { to } 10 \text { days after treatment may cause injury. Do } \\
\text { NOT add wetting agents or surfactants to the spray solution. }\end{array}$ \\
\hline $\begin{array}{l}\text { Buctril 2L } \\
1.5 \mathrm{pt}\end{array}$ & bromoxynil & $\begin{array}{l}\text { Apply to seedling alfalfa that has a minimum of four trifoliolate } \\
\text { leaves. Spray winter annual broadleaf weeds that do not exceed the } \\
\text { 4-leaf stage or } 2 \text { inches in height, or have rosettes greater than } 1.5 \\
\text { inches in diameter. DO NOT apply when temperatures exceed } 70^{\circ} \mathrm{F} \text {. } \\
\text { at and } 3 \text { days after application or unacceptable crop injury may } \\
\text { occur. DO NOT add a surfactant or crop oil. DO NOT cut for feed or } \\
\text { graze spring treated alfalfa for } 30 \text { days after application. DO NOT cut } \\
\text { for feed or graze fall or winter treated alfalfa for } 60 \text { days after } \\
\text { application. }\end{array}$ \\
\hline
\end{tabular}

1. This document is SS-AGR-229, one of a series of the Agronomy Department, Florida Cooperative Extension Service, Institute of Food and Agricultural Sciences, University of Florida. Published January 2005. Visit the EDIS Web Site at http://edis.ifas.ufl.edu.

2. J.A. Ferrell, assistant professor, Agronomy Department; Florida Cooperative Extension Service, Institute of Food and Agricultural Sciences, University of Florida, Gainesville, FL 32611.

The use of trade names in this publication is solely for the purpose of providing specific information. UF/IFAS does not guarantee or warranty the products named, and references to them in this publication does not signify our approval to the exclusion of other products of suitable composition. Use herbicides safely. Read and follow directions on the manufacturer's label.

The Institute of Food and Agricultural Sciences is an equal opportunity/affirmative action employer authorized to provide research, educational information and other services only to individuals and institutions that function without regard to race, color, sex, age, handicap, or national origin. For information on obtaining other extension publications, contact your county Cooperative Extension Service office. Florida Cooperative Extension Service/Institute of Food and Agricultural Sciences/University of Florida/Christine Taylor Waddill, Dean. 
Table 1. Weed Management in Alfalfa.

\begin{tabular}{|c|c|c|}
\hline $\begin{array}{l}\text { Trade Name and } \\
\text { Broadcast Rate/Acre of } \\
\text { Commercial Product }\end{array}$ & Common Name & Remarks \\
\hline $\begin{array}{l}\text { Pursuit } 70 \mathrm{DG} \\
1.08 \text { to } 2.16 \mathrm{oz} .\end{array}$ & imazethapyr & $\begin{array}{l}\text { Apply to seedling alfalfa with a minimum of two trifoliate leaves and } \\
\text { when weeds are } 1 \text { to } 3 \text { inches tall or before rosette forming weeds } \\
\text { exceed } 3 \text { inches in diameter. Pursuit requires a } 1 \text {-hour rain free } \\
\text { period. Pursuit may cause a temporary reduction in height or slight } \\
\text { leaf yellowing. DO NOT feed, graze, or harvest alfalfa for } 30 \text { days } \\
\text { after application. A maximum total of } 2.16 \text { oz./A of Pursuit may be } \\
\text { applied per year. Add a nonionic surfactant at } 0.25 \% \mathrm{v} / \mathrm{v} \text { or a crop oil } \\
\text { concentrate at } 1.5 \text { to } 2.0 \text { pts/A to the spray solution. If replanting is } \\
\text { necessary in a field treated with Pursuit, do not plant alfalfa for } 4 \\
\text { months following the application of Pursuit. Do not apply more than } \\
1.44 \text { oz. during the last year of the stand. }\end{array}$ \\
\hline $\begin{array}{l}\text { Raptor } \\
4 \text { to } 6 \mathrm{fl} \mathrm{oz.}\end{array}$ & imazamox & $\begin{array}{l}\text { Apply after the } 2 \text { nd trifoliate stage, or larger, when weeds are } 3 " \text { in } \\
\text { height or less. There may be temporary reduction in growth, but } \\
\text { alfalfa will quickly recover. Add a nonionic surfactant at } 0.25 \% \mathrm{v} / \mathrm{v} \text { or } \\
\text { a crop oil concentrate at } 1 \% \mathrm{v} / \mathrm{v} \text { to the spray solution. DO NOT } \\
\text { harvest alfalfa hay for } 20 \text { days, or graze for } 70 \text { days, after } \\
\text { application. A maximum total of } 6 \mathrm{fl} \text {. oz./A of Raptor may be applied } \\
\text { per year. }\end{array}$ \\
\hline $\begin{array}{l}\text { Kerb } 50 \mathrm{~W} \\
1 \text { to } 1.5 \mathrm{lb}\end{array}$ & pronamid & $\begin{array}{l}\text { Spray preemergence or early postemergence for control of winter } \\
\text { annual weeds after the alfalfa has reached the trifoliolate leaf stage. } \\
\text { Do not graze or cut for hay for } 120 \text { days after treatment. }\end{array}$ \\
\hline $\begin{array}{l}\text { Poast ( } 1.5 \mathrm{lb} \text { gal) } \\
1 \text { to } 2.5 \mathrm{pt} \\
\text { or } \\
\text { Poast Plus ( } 1 \mathrm{lb} \text { gal) } \\
1.5 \text { to } 2.25 \mathrm{pt}\end{array}$ & sethoxydim & $\begin{array}{l}\text { Apply } 1 \text { pt. of Poast or } 1.5 \text { pts. of Poast Plus with } 2 \text { pts. of crop oil } \\
\text { concentrate/A for control of annual grasses up to } 8 \text { inches tall (varies } \\
\text { with species). Use } 21 / 2 \text { pts./A of Poast or } 2.25 \text { pts./A of Poast Plus if } \\
\text { rhizome johnsongrass is to be controlled, followed by a second } \\
\text { application to regrowth or new plants. Do not apply to grass or alfalfa } \\
\text { under stress. Apply before the grass has been cut. Do not apply } \\
\text { Poast or Poast Plus within } 7 \text { days of feeding, grazing or harvesting } \\
\text { undried forage, or within } 14 \text { days of cutting for dry hay. }\end{array}$ \\
\hline $\begin{array}{l}\text { Select 2EC } \\
\text { or } \\
\text { Arrow } \\
6 \text { to } 8 \mathrm{fl} \mathrm{oz.}\end{array}$ & clethodim & $\begin{array}{l}\text { Apply with a crop oil concentrate at } 1 \% \mathrm{v} / \mathrm{v} \text { for the control of most } \\
\text { annual grasses up to } 6 \text { to } 8 \text { inches tall. Also, has good activity on } \\
\text { johnsongrass and suppresses common bermudagrass. DO NOT } \\
\text { apply within } 15 \text { days of grazing, feeding, or harvesting alfalfa for } \\
\text { forage or hay. May be tank-mixed with } 2,4-\mathrm{DB} \text {; however, the risk of } \\
\text { temporary injury to alfalfa will increase. }\end{array}$ \\
\hline \multicolumn{3}{|c|}{ Postemergence - Seedling (Between Cuttings) } \\
\hline $\begin{array}{l}\text { Raptor } \\
4 \text { to } 6 \mathrm{fl} \mathrm{oz.}\end{array}$ & imazamox & $\begin{array}{l}\text { Apply when weeds are } 3 " \text { in height or less. See above for additional } \\
\text { surfactant and grazing information. }\end{array}$ \\
\hline $\begin{array}{l}\text { Gramoxone Max } \\
0.7 \mathrm{pt}\end{array}$ & paraquat & $\begin{array}{l}\text { Apply to small emerged annual grass and broadleaf weeds in first } \\
\text { year alfalfa immediately after removing hay between cuttings. Do not } \\
\text { treat more than } 5 \text { days after cutting. Add a nonionic surfactant at } 1 \\
\text { pt./ } 100 \text { gals. of water. Do not make more than two applications } \\
\text { during the growing season. Apply in } 20-40 \text { gals. of water/A. Weeds } \\
\text { much beyond the seedling stage and stubble of those cut off during } \\
\text { harvest will be less affected by the treatment. Do not graze, cut or } \\
\text { harvest within } 30 \text { days of application. }\end{array}$ \\
\hline
\end{tabular}


Table 1. Weed Management in Alfalfa.

\begin{tabular}{|c|c|c|}
\hline $\begin{array}{l}\text { Trade Name and } \\
\text { Broadcast Rate/Acre of } \\
\text { Commercial Product }\end{array}$ & Common Name & Remarks \\
\hline \multicolumn{3}{|c|}{ Postemergence - Established Dormant Alfalfa (greater than 1 year old) } \\
\hline $\begin{array}{l}\text { Sencor } 4 \mathrm{~L} \\
12 \mathrm{fl} \mathrm{oz} \\
\text { Sencor 75DF } \\
\text { or } \\
\text { Lexone 75DF } \\
8 \mathrm{oz}\end{array}$ & metribuzin & \multirow{2}{*}{$\begin{array}{l}\text { See label for weeds controlled at different rates and for precautions } \\
\text { related to soil textures. Spray dormant alfalfa, established one year } \\
\text { or longer, after frost in fall or preferably in late winter before alfalfa } \\
\text { begins spring growth while weeds are small. Provides preemergence } \\
\text { and early postemergence weed control when surfactant is added. } \\
\text { Do not use these treatments on alfalfa-grass mixtures. Do not use } \\
\text { Sencor, Lexone or Velpar on sands. The higher rate of Velpar may } \\
\text { cause crop damage. Do not graze or cut for hay within } 28 \text { days after } \\
\text { Sencor or Lexone treatment. Do not graze or cut for hay within } 30 \\
\text { days after Velpar treatment. Do not tank-mix any of these herbicides. }\end{array}$} \\
\hline $\begin{array}{l}\text { Velpar } 2 \mathrm{~L} \\
2 \text { to } 3 \mathrm{pt} \\
\text { or } \\
\text { Velpar } 90 \mathrm{SP} \\
8 \text { to } 12 \mathrm{oz}\end{array}$ & hexazinone & \\
\hline $\begin{array}{l}\text { Kerb } 50 \mathrm{~W} \\
1 \text { to } 1.5 \mathrm{lb}\end{array}$ & pronamide & $\begin{array}{l}\text { Apply in January at lower rate for most annual winter grasses, except } \\
\text { higher rate for annual ryegrass or orchardgrass. Do not graze or cut } \\
\text { for hay for } 120 \text { days after treatment. Provides preemergence and } \\
\text { early postemergence control. Do not tank mix with other } \\
\text { herbicides. }\end{array}$ \\
\hline $\begin{array}{l}\text { Raptor } \\
4 \text { to } 6 \mathrm{fl} \mathrm{oz.}\end{array}$ & imazamox & $\begin{array}{l}\text { Apply when weeds are } 3 " \text { in height or less. See above for additional } \\
\text { surfactant and grazing information. }\end{array}$ \\
\hline $\begin{array}{l}\text { Gramoxone Max } \\
0.7 \text { to } 1.3 \mathrm{pt}\end{array}$ & paraquat & $\begin{array}{l}\text { Apply during the winter months when established alfalfa is dormant. } \\
\text { DO NOT apply dormant treatments to seedling alfalfa less than } \\
6 \text { months old. Controls Italian ryegrass (less than } 6 \text { in. tall), } \\
\text { chickweed and most other winter annual weeds. Green alfalfa foliage } \\
\text { present at time of application will become necrotic (brown). DO NOT } \\
\text { apply after the initiation of new spring growth. Add a nonionic } \\
\text { surfactant at } 1.0 \text { pt./100 gals. of spray solution. DO NOT graze or } \\
\text { harvest within } 60 \text { days of application. Make only one application } \\
\text { per season. }\end{array}$ \\
\hline \multicolumn{3}{|c|}{ Preemergence - Established Alfalfa } \\
\hline $\begin{array}{l}\text { Zorial Rapid } 80 \text { (80 DG) } \\
1.25 \text { to } 2.5 \mathrm{lb}\end{array}$ & norflurazon & $\begin{array}{l}\text { Apply to established alfalfa in spring or early fall for the control of } \\
\text { annual grasses and annual broadleaf weeds such as tropic croton } \\
\text { and prickly sida. May be applied to dormant and actively-growing } \\
\text { alfalfa. Apply to actively-growing alfalfa following hay removal to } \\
\text { ensure spray penetration to the soil surface. DO NOT apply to } \\
\text { seedling alfalfa earlier than } 5 \text { months after emergence. DO NOT } \\
\text { apply Zorial Rapid } 80 \text { within } 28 \text { days of harvest. Use the low rate on } \\
\text { sandy soils. Zorial Rapid } 80 \text { may be tank-mized with Gramoxone } \\
\text { Extra, Poast, Kerb, Sencor, Lexone and } 2,4-D B \text {. For } 16 \text { months } \\
\text { following application rotate only to cotton, soybeans or peanuts. }\end{array}$ \\
\hline \multicolumn{3}{|c|}{ Postemergence - Established Alfalfa } \\
\hline $\begin{array}{l}\text { Butyrac } 200 \\
\text { or } \\
\text { Butoxone } \\
2 \text { to } 3 \text { qts }\end{array}$ & 2,4-DB & $\begin{array}{l}\text { Spray established dormant or non-dormant alfalfa in late fall through } \\
\text { spring for control of emerged weeds that emerge in the fall and over } \\
\text { winter in the rosette stage. Do not graze or cut for hay within } 30 \text { days } \\
\text { after treatment. Overhead irrigation or rainfall within a few days after } \\
\text { use may wash chemical into the root zone possibly causing some } \\
\text { twisting of stems and malformation of leaves. Do not add wetting } \\
\text { agents or surfactants to the spray mix. }\end{array}$ \\
\hline
\end{tabular}


Table 1. Weed Management in Alfalfa.

\begin{tabular}{|c|c|c|}
\hline $\begin{array}{l}\text { Trade Name and } \\
\text { Broadcast Rate/Acre of } \\
\text { Commercial Product }\end{array}$ & Common Name & Remarks \\
\hline $\begin{array}{l}\text { Pursuit } 70 \mathrm{DG} \\
1.08 \text { to } 2.16 \mathrm{oz}\end{array}$ & imazethapyr & $\begin{array}{l}\text { Apply to established alfalfa in the fall, or in the spring to dormant or } \\
\text { semi-dormant alfalfa. Spring treatments should be made before } \\
\text { excessive alfalfa growth (less than } 3 \text { inches of new growth) to reduce } \\
\text { spray interference. Apply when weeds are } 1 \text { to } 3 \text { inches tall or before } \\
\text { rosette forming weeds exceed } 3 \text { inches in diameter. DO NOT feed, } \\
\text { graze, or harvest alfalfa for } 30 \text { days after application. A maximum } \\
\text { total of } 2.16 \text { oz./A of Pursuit may be applied per year. Add a nonionic } \\
\text { surfactant at } 0.25 \% \text { v/v or a crop oil concentrate at } 1.5 \text { to } 2.0 \text { pts/A to } \\
\text { the spray solution. If replanting is necessary in a field treated with } \\
\text { Pursuit, do not plant alfalfa for } 4 \text { months following the application of } \\
\text { Pursuit. Do not apply more than } 1.44 \text { oz. during the last year of the } \\
\text { stand. }\end{array}$ \\
\hline $\begin{array}{l}\text { Raptor } \\
4 \text { to } 6 \mathrm{fl} \mathrm{oz.}\end{array}$ & imazamox & $\begin{array}{l}\text { Apply when weeds are } 3 " \text { in height or less. See above for additional } \\
\text { surfactant and grazing information. }\end{array}$ \\
\hline $\begin{array}{l}\text { Poast (1.5 lb gal) } \\
1 \text { to } 2.5 \mathrm{pt} \\
\text { or } \\
\text { Poast Plus ( } 1 \mathrm{lb} \text { gal) } \\
1.5 \text { to } 2.25 \mathrm{pt}\end{array}$ & sethoxydim & $\begin{array}{l}\text { Apply } 1 \text { pt. of Poast or } 1.5 \text { pts. of Poast Plus with } 2 \text { pts. of crop oil } \\
\text { concentrate/A for control of annual grasses up to } 8 \text { inches tall (varies } \\
\text { with species). Use } 21 / 2 \text { pts./A of Poast or } 2.25 \text { pts./A of Poast Plus if } \\
\text { rhizome johnsongrass is to be controlled, followed by a second } \\
\text { application to regrowth or new plants. Do not apply to grass or alfalfa } \\
\text { under stress. Apply before the grass has been cut. Do not apply } \\
\text { Poast or Poast Plus within } 7 \text { days of feeding, grazing or harvesting } \\
\text { undried forage, or within } 14 \text { days of cutting for dry hay. }\end{array}$ \\
\hline $\begin{array}{l}\text { Select 2EC } \\
\text { or } \\
\text { Arrow } \\
6 \text { to } 8 \mathrm{fl} \mathrm{oz.}\end{array}$ & clethodim & $\begin{array}{l}\text { Apply with a crop oil concentrate at } 1 \% \mathrm{v} / \mathrm{v} \text { for the control of most } \\
\text { annual grasses up to } 6 \text { to } 8 \text { inches tall. Also, has good activity on } \\
\text { johnsongrass and suppresses common bermudagrass. DO NOT } \\
\text { apply within } 15 \text { days of grazing, feeding, or harvesting alfalfa for } \\
\text { forage or hay. May be tank-mixed with } 2,4-D B \text {; however, the risk of } \\
\text { temporary injury to alfalfa will increase. }\end{array}$ \\
\hline \multicolumn{3}{|c|}{ Postemergence - Established Alfalfa (Between Cuttings) } \\
\hline $\begin{array}{l}\text { Pursuit } 70 \mathrm{DG} \\
1.08 \text { to } 2.16 \mathrm{oz}\end{array}$ & imazethapyr & $\begin{array}{l}\text { Apply as a between cut treatment. Remove hay from the field and } \\
\text { apply before excessive alfalfa regrowth. Apply when weeds are } 1 \text { to } \\
3 \text { inches tall or before rosette forming weeds exceed } 3 \text { inches in } \\
\text { diameter.DO NOT feed, graze, or harvest alfalfa for } 30 \text { days after } \\
\text { application. A maximum total of } 2.16 \mathrm{oz} . / \mathrm{A} \text { of Pursuit may be applied } \\
\text { per year. Add a nonionic surfactant at } 0.25 \% \text { v/v or a crop oil } \\
\text { concentrate at } 1.5 \text { to } 2.0 \text { pts/A to the spray solution. If replanting is } \\
\text { necessary in a field treated with Pursuit, do not plant alfalfa for } 4 \\
\text { months following the application of Pursuit. Do not apply more than } \\
1.44 \text { oz. during the last year of the stand. }\end{array}$ \\
\hline $\begin{array}{l}\text { Raptor } \\
4 \text { to } 6 \mathrm{fl} \mathrm{oz.}\end{array}$ & imazamox & $\begin{array}{l}\text { Apply when weeds are } 3 " \text { in height or less. See above for additional } \\
\text { surfactant and grazing information. }\end{array}$ \\
\hline $\begin{array}{l}\text { Gramoxone Max } \\
0.7 \mathrm{pt}\end{array}$ & paraquat & $\begin{array}{l}\text { Apply to established stands (at least one year old) immediately after } \\
\text { alfalfa has been removed for silage or hay. Do not treat more than } 5 \\
\text { days after cutting. Add surfactant at } 1 \text { pt./100 gals. of spray mix. DO } \\
\text { NOT graze, cut or harvest within } 30 \text { days of application. Make } 1 \text { to } 3 \\
\text { applications as needed during the cutting season. Apply in } 20 \text { to } 40 \\
\text { gals. of water/A. Weeds much beyond the seedling stage and stubble } \\
\text { of those cut off during harvest will be less affected by the treatment. }\end{array}$ \\
\hline
\end{tabular}


Table 2. Estimated effectiveness of recommended herbicides on common weeds in Florida Alfalfa.

\begin{tabular}{|c|c|c|c|c|c|}
\hline \multirow[t]{2}{*}{ Time of Application } & PPI & PPI & PRE & POT & POT \\
\hline & Balan & EPTC - Eptam & Kerb & $\begin{array}{c}\text { Poast or } \\
\text { Select }\end{array}$ & $\begin{array}{c}\text { Pursuit or } \\
\text { Raptor }\end{array}$ \\
\hline bahiagrass & $\mathrm{P}$ & $P$ & $P$ & $\mathrm{~F}$ & -- \\
\hline bermudagrass & $P$ & $P$ & $P$ & $F-G$ & $P$ \\
\hline bitter sneezeweed & $\mathrm{P}$ & $\mathrm{P}$ & $\mathrm{P}$ & $\mathrm{P}$ & -- \\
\hline blackberry & $P$ & $P$ & $P$ & $P$ & -- \\
\hline bracken fern & $P$ & $P$ & $\mathrm{P}$ & $\mathrm{P}$ & -- \\
\hline briars (Smilax) & $P$ & $P$ & $P$ & $P$ & -- \\
\hline broomsedge & $P$ & $P$ & $P$ & $P$ & -- \\
\hline bulrush & $P$ & $P$ & $P$ & $P$ & -- \\
\hline buttercup & $P$ & $P$ & $P$ & $P$ & -- \\
\hline chickweed & $\mathrm{F}$ & $E$ & $\mathrm{G}$ & $\mathrm{P}$ & $\mathrm{E}$ \\
\hline crabgrass & $E$ & $\mathrm{G}$ & $\mathrm{F}$ & G-E & $\mathrm{F}$ \\
\hline cudweed & $P$ & $\mathrm{P}$ & $P$ & $\mathrm{P}$ & -- \\
\hline curly dock & $\mathrm{P}$ & $\mathrm{P}$ & $\mathrm{P}$ & $\mathrm{P}$ & $\mathrm{F}$ \\
\hline dallisgrass & $\mathrm{P}$ & $\mathrm{P}$ & $\mathrm{P}$ & $\mathrm{P}$ & -- \\
\hline dandelion & $P$ & $\mathrm{G}$ & $P$ & $P$ & -- \\
\hline dodder & $\mathrm{P}$ & $\mathrm{P}$ & $\mathrm{E}$ & $\mathrm{P}$ & -- \\
\hline dogfennel & $P$ & $P$ & $P$ & $P$ & -- \\
\hline evening primrose & $\mathrm{F}$ & $F-G$ & $P$ & $P$ & -- \\
\hline foxtails & $\mathrm{G}$ & $\mathrm{G}$ & $P$ & $\mathrm{E}$ & $\mathrm{G}$ \\
\hline gallberry & $\mathrm{P}$ & $\mathrm{P}$ & $P$ & $P$ & -- \\
\hline goldenrod & $P$ & $P$ & $P$ & $P$ & -- \\
\hline henbit & $\mathrm{F}$ & $\mathrm{G}$ & $P$ & $P$ & $E$ \\
\hline honeysuckle & $P$ & $P$ & $P$ & $P$ & -- \\
\hline horsenettle & $P$ & $P$ & $P$ & $\mathrm{P}$ & -- \\
\hline horseweed & $\mathrm{P}$ & $\mathrm{P}$ & $\mathrm{P}$ & $\mathrm{P}$ & -- \\
\hline Italian ryegrass & $\mathrm{G}$ & $E$ & $\mathrm{G}$ & $E$ & $P$ \\
\hline johnsongrass & $\mathrm{P}$ & $\mathrm{P}$ & $\mathrm{P}$ & G & $\mathrm{P}$ \\
\hline kudzu & $\mathrm{P}$ & $P$ & $P$ & $P$ & -- \\
\hline little barley & $\mathrm{G}$ & $\mathrm{G}$ & $E$ & $\mathrm{~F}$ & -- \\
\hline mayweed & -- & -- & $P$ & $P$ & -- \\
\hline nettle, stinging & $P$ & $P$ & $P$ & $\mathrm{P}$ & -- \\
\hline nutsedge & $P$ & $\mathrm{~F}$ & $P$ & $\mathrm{P}$ & $\mathrm{F}$ \\
\hline palmetto & $\mathrm{P}$ & $P$ & $P$ & $\mathrm{P}$ & -- \\
\hline perilla mint & $P$ & $P$ & -- & $P$ & -- \\
\hline persimmon & $\mathrm{P}$ & $P$ & $P$ & $\mathrm{P}$ & -- \\
\hline pigweed species & $\mathrm{G}$ & G & -- & $\mathrm{P}$ & $\mathrm{G}$ \\
\hline pokeberry & $\mathrm{P}$ & $P$ & $P$ & $\mathrm{P}$ & -- \\
\hline prickly pear & $P$ & $P$ & $P$ & $P$ & -- \\
\hline ragweed & $P$ & $P$ & $P$ & $\mathrm{P}$ & $\mathrm{F}$ \\
\hline red sorrel & $\mathrm{P}$ & $\mathrm{P}$ & $\mathrm{P}$ & $\mathrm{P}$ & -- \\
\hline sandbur & $E$ & $\mathrm{G}$ & $P$ & $\mathrm{G}$ & -- \\
\hline shepherds-purse & $\mathrm{P}$ & $\mathrm{G}$ & $\mathrm{G}$ & $P$ & $\mathrm{E}$ \\
\hline sicklepod & $P$ & $\mathrm{~F}$ & $P$ & $P$ & -- \\
\hline smutgrass & $\mathrm{P}$ & $\mathrm{P}$ & $\mathrm{P}$ & $\mathrm{P}$ & -- \\
\hline
\end{tabular}


Table 2. Estimated effectiveness of recommended herbicides on common weeds in Florida Alfalfa.

\begin{tabular}{||l|c|c|c|c|c||}
\hline \hline \multirow{3}{*}{ Time of Application } & PPI & PPI & PRE & POT & POT \\
\cline { 2 - 6 } & Balan & EPTC - Eptam & Kerb & $\begin{array}{c}\text { Poast or } \\
\text { Select }\end{array}$ & $\begin{array}{c}\text { Pursuit or } \\
\text { Raptor }\end{array}$ \\
\hline swinecress & $\mathrm{P}$ & $\mathrm{G}$ & $\mathrm{F}$ & $\mathrm{P}$ & -- \\
\hline Texas panicum & $\mathrm{G}-\mathrm{E}$ & $\mathrm{G}$ & $\mathrm{P}$ & $\mathrm{E}$ & -- \\
\hline thistles & $\mathrm{P}$ & $\mathrm{E}$ & $\mathrm{P}$ & $\mathrm{P}$ & $\mathrm{P}$ \\
\hline tropical soda apple & $\mathrm{P}$ & $\mathrm{P}$ & $\mathrm{P}$ & $\mathrm{P}$ & -- \\
\hline vaseygrass & $\mathrm{P}$ & $\mathrm{P}$ & $\mathrm{P}$ & $\mathrm{P}$ & -- \\
\hline Virginia pepperweed & $\mathrm{P}$ & $\mathrm{G}$ & $\mathrm{P}$ & $\mathrm{P}$ & -- \\
\hline wax myrtle & $\mathrm{P}$ & $\mathrm{P}$ & $\mathrm{P}$ & $\mathrm{P}$ & -- \\
\hline wild cherry & $\mathrm{P}$ & $\mathrm{P}$ & $\mathrm{P}$ & $\mathrm{P}$ & -- \\
\hline wild garlic & $\mathrm{P}$ & $\mathrm{P}$ & $\mathrm{P}$ & $\mathrm{P}$ & -- \\
\hline wild radish & $\mathrm{P}$ & $\mathrm{P}-\mathrm{F}$ & $\mathrm{P}$ & $\mathrm{P}$ & $\mathrm{G}-\mathrm{E}$ \\
\hline wild plum & $\mathrm{P}$ & $\mathrm{P}$ & $\mathrm{P}$ & $\mathrm{P}$ & -- \\
\hline wild rose & $\mathrm{P}$ & $\mathrm{P}$ & $\mathrm{P}$ & $\mathrm{P}$ & -- \\
\hline wooly croton & $\mathrm{P}$ & $\mathrm{P}$ & $\mathrm{P}$ & $\mathrm{P}$ & $\mathrm{P}$ \\
\hline \hline
\end{tabular}

Table 3. Estimated effectiveness of recommended herbicides on common weeds in Florida Alfalfa (continued).

\begin{tabular}{||l|c|c|c|c|c|c||}
\hline \hline $\begin{array}{l}\text { Time of } \\
\text { Application }\end{array}$ & POT & POT & POT & POT & POT & POT \\
\hline & Velpar & $\begin{array}{c}\text { Sencor or } \\
\text { Lexone }\end{array}$ & $\begin{array}{c}\text { Paraquat- } \\
\text { Gramoxone Max }\end{array}$ & Buctril & 2,4-D & 2,4-DB \\
\hline bahiagrass & $\mathrm{P}$ & $\mathrm{P}$ & $\mathrm{P}$ & $\mathrm{P}$ & $\mathrm{P}$ & $\mathrm{P}$ \\
\hline bermudagrass & $\mathrm{P}$ & $\mathrm{P}$ & $\mathrm{P}$ & $\mathrm{P}$ & $\mathrm{P}$ & $\mathrm{P}$ \\
\hline bitter sneezeweed & -- & -- & -- & $\mathrm{E}$ & $\mathrm{G}$ \\
\hline blackberry & $\mathrm{F}$ & $\mathrm{P}$ & $\mathrm{P}$ & $\mathrm{P}$ & $\mathrm{F}$ & $\mathrm{P}$ \\
\hline bracken fern & $\mathrm{F}$ & -- & $\mathrm{P}$ & $\mathrm{P}$ & $\mathrm{P}$ \\
\hline briars (Smilax) & $\mathrm{F}$ & $\mathrm{P}$ & $\mathrm{P}$ & $\mathrm{P}$ & $\mathrm{P}$ & $\mathrm{P}$ \\
\hline broomsedge & $\mathrm{P}$ & $\mathrm{P}$ & $\mathrm{P}$ & $\mathrm{P}$ & $\mathrm{P}$ & $\mathrm{P}$ \\
\hline bulrush & -- & -- & $\mathrm{P}$ & $\mathrm{P}$ & $\mathrm{P}$ \\
\hline buttercup & $\mathrm{G}$ & $\mathrm{G}$ & $\mathrm{G}$ & -- & $\mathrm{E}$ & $\mathrm{F}$ \\
\hline chickweed & $\mathrm{E}$ & $\mathrm{E}$ & $\mathrm{E}$ & $\mathrm{F}$ & $\mathrm{F}$ & $\mathrm{P}$ \\
\hline crabgrass & $\mathrm{P}$ & $\mathrm{F}$ & $\mathrm{F}$ & $\mathrm{P}$ & $\mathrm{P}-\mathrm{F}$ & $\mathrm{P}$ \\
\hline cudweed & -- & -- & $\mathrm{G}$ & $\mathrm{P}$ & $\mathrm{F}$ & -- \\
\hline curly dock & $\mathrm{P}$ & -- & $\mathrm{P}$ & -- & $\mathrm{F}$ & $\mathrm{P}$ \\
\hline dallisgrass & -- & $\mathrm{P}$ & $\mathrm{P}$ & $\mathrm{P}$ & $\mathrm{P}$ & $\mathrm{P}$ \\
\hline dandelion & $\mathrm{E}$ & $\mathrm{G}$ & $\mathrm{G}$ & $\mathrm{P}$ & $\mathrm{E}$ & $\mathrm{G}$ \\
\hline dodder & -- & -- & $\mathrm{G}-\mathrm{E}$ & -- & $\mathrm{P}$ & $\mathrm{P}$ \\
\hline dogfennel & -- & $\mathrm{P}$ & $\mathrm{P}$ & $\mathrm{F}$ & $\mathrm{P}$ \\
\hline evening primrose & $\mathrm{E}$ & $\mathrm{G}$ & $\mathrm{P}-\mathrm{F}$ & -- & $\mathrm{E}$ & $\mathrm{G}$ \\
\hline foxtails & -- & $\mathrm{P}$ & $\mathrm{F}$ & $\mathrm{P}$ & $\mathrm{P}$ & $\mathrm{P}$ \\
\hline gallberry & $\mathrm{P}$ & $\mathrm{P}$ & $\mathrm{P}$ & $\mathrm{P}$ & $\mathrm{G}$ & $\mathrm{P}$ \\
\hline goldenrod & -- & $\mathrm{P}$ & $\mathrm{P}$ & $\mathrm{F}$ & $\mathrm{P}$ \\
\hline henbit & $\mathrm{E}$ & $\mathrm{E}$ & $\mathrm{G}$ & $\mathrm{F}$ & $\mathrm{F}$ & $\mathrm{P}$ \\
\hline honeysuckle & -- & $\mathrm{P}$ & $\mathrm{P}$ & $\mathrm{P}$ & $\mathrm{E}$ & $\mathrm{P}$ \\
\hline horsenettle & -- & $\mathrm{P}$ & $\mathrm{P}$ & $\mathrm{P}$ & $\mathrm{P}$ & $\mathrm{P}$ \\
\hline \hline
\end{tabular}


Table 3. Estimated effectiveness of recommended herbicides on common weeds in Florida Alfalfa (continued).

\begin{tabular}{|c|c|c|c|c|c|c|}
\hline $\begin{array}{l}\text { Time of } \\
\text { Application }\end{array}$ & РOT & РОТ & РОт & РОт & РОТ & РОT \\
\hline & Velpar & $\begin{array}{c}\text { Sencor or } \\
\text { Lexone }\end{array}$ & $\begin{array}{c}\text { Paraquat- } \\
\text { Gramoxone Max }\end{array}$ & Buctril & 2,4-D & 2,4-DB \\
\hline horseweed & -- & $\mathrm{P}$ & $\mathrm{P}$ & $\mathrm{P}$ & $\mathrm{F}$ & $\mathrm{P}$ \\
\hline Italian ryegrass & $\mathrm{G}$ & $P$ & G-E & $P$ & $P$ & $P$ \\
\hline johnsongrass & -- & $P$ & $\mathrm{P}$ & $P$ & $P$ & $P$ \\
\hline kudzu & -- & $\mathrm{P}$ & $\mathrm{P}$ & $P$ & $\mathrm{P}-\mathrm{F}$ & $P$ \\
\hline little barley & $E$ & $P$ & G-E & $P$ & $\mathrm{P}$ & $P$ \\
\hline mayweed & $F-G$ & -- & $\mathrm{E}$ & -- & $\mathrm{F}$ & $P$ \\
\hline nettle, stinging & -- & -- & -- & -- & $P$ & $P$ \\
\hline nutsedge & $P$ & $P$ & $P$ & $P$ & $P$ & $P$ \\
\hline palmetto & $P$ & $P$ & $P$ & $P$ & $P$ & $P$ \\
\hline perilla mint & -- & -- & -- & -- & $P$ & -- \\
\hline persimmon & $\mathrm{F}$ & $P$ & $P$ & $P$ & $P$ & $P$ \\
\hline pigweed & $\mathrm{G}$ & $\mathrm{G}$ & $\mathrm{G}$ & $\mathrm{F}$ & $\mathrm{F}$ & G \\
\hline pokeberry & -- & -- & -- & $P$ & $E$ & $\mathrm{G}$ \\
\hline prickly pear & $P$ & $P$ & $P$ & $P$ & $P$ & $P$ \\
\hline ragweed & $\mathrm{F}$ & $\mathrm{G}$ & $\mathrm{G}$ & $\mathrm{F}$ & $E$ & $\mathrm{G}$ \\
\hline red sorrel & -- & -- & $\mathrm{P}-\mathrm{F}$ & -- & $P$ & $\mathrm{P}$ \\
\hline sandbur & -- & $\mathrm{F}$ & G & $\mathrm{P}$ & $\mathrm{P}$ & $\mathrm{P}$ \\
\hline $\begin{array}{l}\text { shepherds- } \\
\text { purse }\end{array}$ & $E$ & $E$ & $\mathrm{G}$ & $\mathrm{G}$ & $E$ & G \\
\hline sicklepod & -- & $\mathrm{F}$ & $F-G$ & -- & $\mathrm{G}$ & $\mathrm{F}$ \\
\hline smutgrass & $\mathrm{G}$ & $P$ & $\mathrm{P}$ & $\mathrm{P}$ & $\mathrm{P}$ & $P$ \\
\hline swinecress & $E$ & $E$ & $E$ & $E$ & $\mathrm{E}$ & $\mathrm{F}$ \\
\hline Texas panicum & -- & P-F & $\mathrm{G}$ & $P$ & $P$ & $P$ \\
\hline thistles & E & G & G & $\mathrm{P}$ & $\mathrm{E}$ & $\mathrm{F}$ \\
\hline $\begin{array}{l}\text { tropical soda } \\
\text { apple }\end{array}$ & $\mathrm{G}$ & $P$ & $P$ & $P$ & P-F & $P$ \\
\hline vaseygrass & -- & $P$ & $P$ & $\mathrm{P}$ & $\mathrm{P}$ & $\mathrm{P}$ \\
\hline $\begin{array}{l}\text { Virginia } \\
\text { pepperweed }\end{array}$ & E & $\mathrm{G}$ & $\mathrm{G}$ & $\mathrm{G}$ & $\mathrm{G}$ & E \\
\hline wax myrtle & $\mathrm{P}$ & $\mathrm{P}$ & $\mathrm{P}$ & $\mathrm{P}$ & G & $\mathrm{P}$ \\
\hline wild cherry & E & $\mathrm{P}$ & $\mathrm{P}$ & $\mathrm{P}$ & E & $\mathrm{P}$ \\
\hline wild garlic & -- & $P$ & $E$ & $\mathrm{P}$ & G-E & $P$ \\
\hline wild radish & $E$ & $E$ & $P$ & $F-G$ & $\mathrm{G}$ & $P$ \\
\hline wild plum & $E$ & $P$ & $P$ & $\mathrm{P}$ & $E$ & $P$ \\
\hline wild rose & -- & $P$ & $P$ & $P$ & $\mathrm{G}$ & $P$ \\
\hline wooly croton & $\mathrm{P}$ & $\mathrm{P}$ & $\mathrm{P}$ & $\mathrm{P}$ & G & $\mathrm{P}$ \\
\hline
\end{tabular}

